\title{
Design and Construction of Infrared Remote Controller for Multiple Home Appliances
}

\author{
Kalayar Win ${ }^{1}$ \& Ni Ni Win ${ }^{2}$ \\ ${ }^{1}$ Department of Engineering Physics, Technological University (Maubin) \\ ${ }^{2}$ Department of Physics, Magway University \\ Lecturer $^{1}$ and Associate Professor ${ }^{2}$ \\ Maubin, Irrawaddy Division, Magway, Magway Division
}

Myanmar

\begin{abstract}
The infrared (IR) remote control devices are used to operate in the most of modern household applications, such as television, $D V D s$, Set top box, Home theatre and air conditioner etc. With the development of the society and smart home, there are more and more home appliances and more infrared remote control devices used to operate them. A single IR remote controller can't be used to manipulate the different kinds of home appliances; as they are not compatible which leads to the wastage of resources. This paper propose an applications and design of Arduino based IR remote control system, which can control multiple devices, code and decode all of the infrared remote control protocol. In this system, we are using IR based wireless communications for controlling home appliances. Arduino is used for controlling whole the process. The remote control transmits a beam of light using an infrared light emitting diode; this light is picked by the IR sensor. After receiving signal from IR remote, Arduino sends related signal to relays which are responsible for switching ON or OFF of the home appliances through a relay driver. The circuit present in this work is designed to control the six electrical devices such as TV, fan, motor, electric bulb, light tube, and CFL to turn on or turn off from a distance using remote control. The program for this project is written in $C$ language and uploaded into the memory of the microcontroller on Arduino board by Arduino IDE window software.
\end{abstract}

Key Words: Arduino UNO, IR Sensor, 20x4 LCD Display, Relay.

\section{INTRODUCTION}

Most homes make use of many electrical and electronics appliances such as the television set, standing fan, ceiling fan, air conditioner, lighting bulbs, video players, radio, etc. All these appliances require switching to turn on and turn off these appliances traditionally. This manual switching of any home appliance is an inconvenient method for physically disabled or elderly or even for the young and busy individuals when frequent switching operation is required. Thus, an easier method of switching is developed to replace this manual switching method, using an advanced switching method for electronic home appliances. Nowadays infrared (IR) remote control system is becoming popular in many applications like switching on/off of televisions, fans, water pumps, air conditioner, lighting bulbs, video players, radio, and more electronic gadgets.

An IR remote works by turning an LED on and off in patterns to produce unique codes. The codes are typically 12 to 32 bits (pieces of data). Each key on the remote is associated with a specific code that is transmitted when the key is pressed. The transmitter in the remote control handset sends out a stream of pulses of infrared light when the user presses a button on the handset. The receiver in the device recognizes the pattern and causes the device to respond accordingly. In this circuit, Arduino UNO board is used as a data receiver and it produces a digital signal (HIGH or LOW) to control the external devices. The Arduino can interact with almost any device that uses some form of remote control, including TVs, audio equipment, cameras, garage doors, appliances, and toys $[1,5]$. 
Arduino responds to IR remote signals using a device called an IR receiver module [3, 4]. This system will demonstrate how to control digital output pins of Arduino using IR remote control. The block diagram of Arduino based home ac appliances control circuit using IR signal is shown in figure 1. LCD is used to display the ON/OFF status of the home ac appliances.

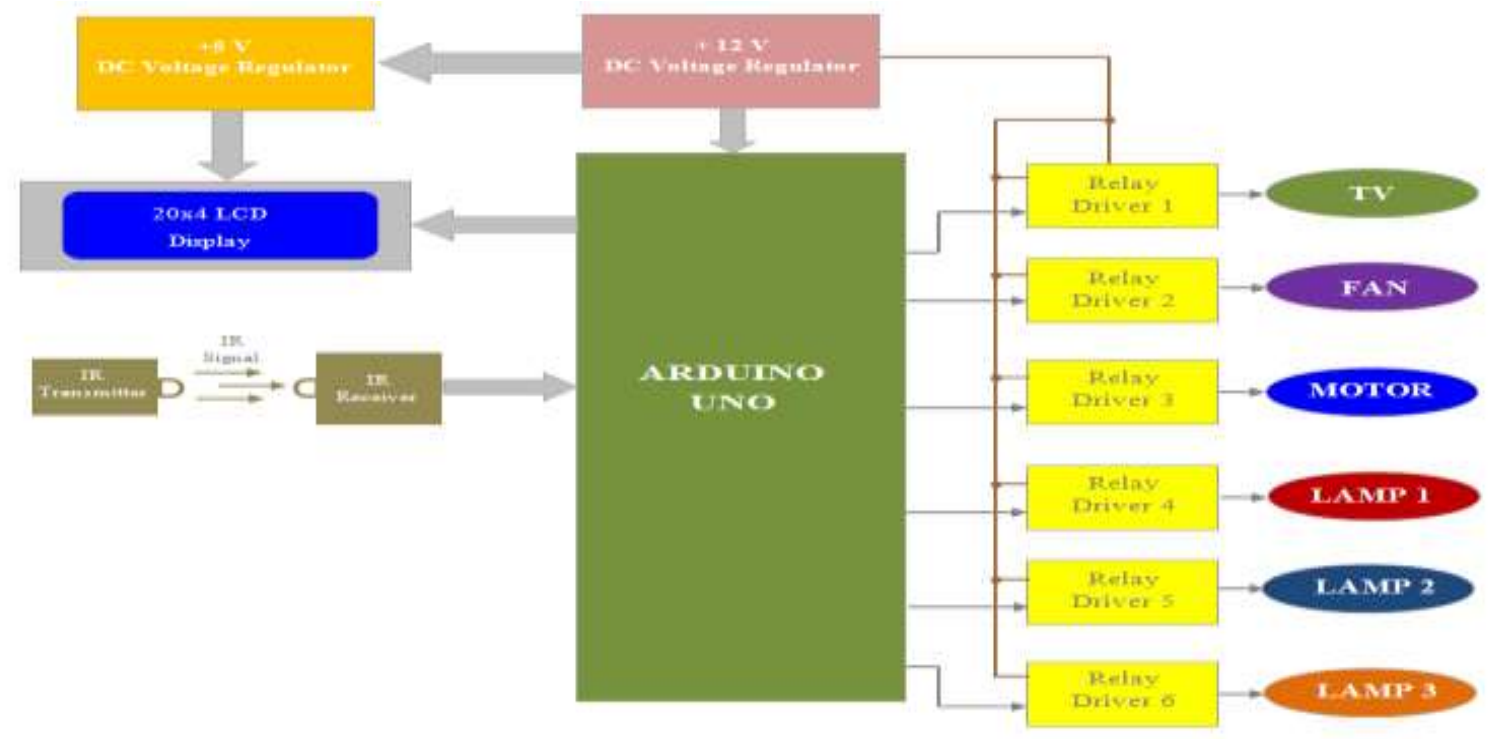

Figure 1. Block diagram of Arduino based home AC appliances control system

\section{INFRARED REMOTE (IR) CONTROL FUNDAMENTAL}

The IR remote is generally used in home theatres and is based on the principle of using infrared as the medium of communication. A TV remote basically consists of a set of buttons and a circuit board. Each button is embedded with a black conductive disk which acts as a contact between the buttons and the printed circuit board. The circuit board or the chip consists of a circuitry to sense the connections or detect the button being pressed and produces the signal in Morse code from which is amplified by the transistors and then given to IR LED [2, 3]. The IR LED is connected to end of the circuit board and emits infrared light which is sensed by the sensor at the receiver of the electrical appliance.

Today's modern remote controls work by modulating the output from an infra-red LED. A series of pulses usually 10-20 pulses of varying width are sent to a gate that turns on and off, the modulator which is usually $38 \mathrm{kHz}$. The reason for modulation is to separate the remote IR range from the IR light emitted by other bodies in the vicinity. Usually it requires a line of sight communication. When a button is pressed, the corresponding circuitry gets connected to bias the IR LED which emits IR light which contains the input. This output is form of light pulses is pulse width modulated at $38 \mathrm{kHz}$ frequency, which is obtained at the receiver by demodulation. In the receiver there is a tone decoder, which responds well to whatever signals the remote sends at a carrier frequency of $38 \mathrm{kHz}$. The microprocessor decodes the series of pulses and determines whether it is valid and if it is, will respond to that function. The receiver at the appliance end generally consists of a TSOP receiver, which receives the IR signal at $38 \mathrm{kHz}$. Basically the sensor senses the IR pulses into electrical signal. This electrical signal is decoded to binary data using a decoder and this binary data is fed to the microprocessor or microcontroller to carry out the required processing of the command being set by pressing the corresponding button. Handheld IR remote control transmitter and receiver [2, 9] use in this research project is shown in figure 2.
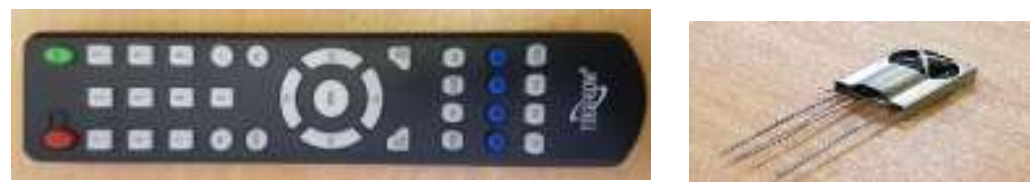

Figure 2. IR remote control Transmitter and receiver

\section{HARDWARE COMPONENTS REQUIRED FOR THE SYSTEM}

\subsection{Arduino Uno}

The Arduino Uno is the most popular one among other Arduino development boards. It is based on the microcontroller ATmega328. Its peripheral features includes 14 digital input/output pins (of which 6 can provide PWM outputs), 6 analog inputs, a $16 \mathrm{MHz}$ crystal oscillator, a USB connector, a power jack, an ICSP header and a reset button. Arduino Uno board can power in 2 
different ways, either by USB connection or by using an external power supply. AC-DC adaptor and battery will come under the category of external power supply. To use battery, connect its leads to the Vin and GND pins on the board. To use adaptor, connect a $2.1 \mathrm{~mm}$ center positive plug in to the board's power jack. The board can operate in the range of 6-20V of the external power source. But the most suitable range is $7-12 \mathrm{~V}$. If the input voltage is less than $7 \mathrm{~V}$, the on-board regulator can't produce proper regulated $5 \mathrm{~V}$ and the board will become unstable. If the input voltage is more than $12 \mathrm{~V}$ it will cause the voltage regulator
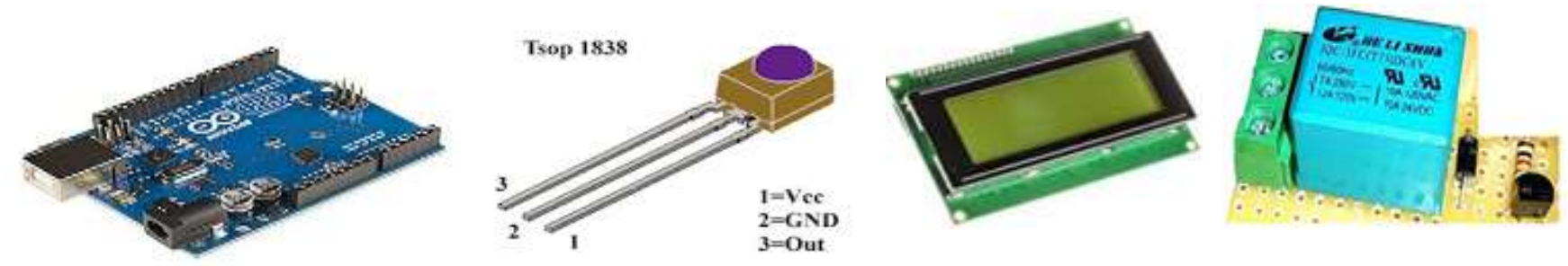

to be overheated and may result in damaging the board. Figure 3 shows an Arduino Uno SMD R3board [3, 6].

Figure 3. Arduino Uno SMD R3board, TSOP 1838 IR receiver, 20x4 alphanumeric LCD and Relay

\subsection{IR LED}

An infrared light emitting diode (IR LED) is a special purpose LED emitting infrared rays ranging $700 \mathrm{~nm}$ to $1 \mathrm{~mm}$ wavelength. Different IR LEDs may produce infrared of differing wavelengths, just like different LEDs produce light of different colours. IR LEDs are usually made of gallium arsenide or aluminium gallium arsenide. In complement with IR receivers, these are commonly used as sensors. The appearance of IR LED is same as a common LED. Since human eye cannot see the infrared radiations, it is not possible for a person to identify if an IR LED is working. A camera on a cell phone camera solves this problem $[2]$.

\subsection{TSOP 1838 IR Infrared Receiver}

The TSOP 1838 series are miniaturized receivers for infrared remote control system. PIN diode and preamplifier are assembled on lead frame, the epoxy package is designed as IR filter. The main benefit is the reliable function even in disturbed ambient and the protection against uncontrolled output pulses. TSOP 1838 infra-red sensor works on the $38 \mathrm{kHz}$ carrier frequency. IR receiver has 3 pins shown in figure 3: $\mathrm{V}_{\mathrm{CC}}$, OUTPUT, and GROUND. Internal circuit of TSOP sensor consists of a photo diode, preamplifier and band pass filter. Photo diode detects the IR rays and amplified signal is passed through the band pass filter. Carrier frequency of the band pass filter (e.g. $38 \mathrm{kHz}$ ). The output of TSOP is active low and it gives $+5 \mathrm{~V}$ in off state. When IR light with a center frequency of $38 \mathrm{kHz}$ incident on TSOP 1838, its output goes low. Specifications of the IR sensor TSOP1838 are: Supply voltage : 2.7 to $5.5 \mathrm{~V}$, Reception Distance : 18 meters, B.P,F Center Frequency :38 kHz, Supply Current : 0.4 to $1.5 \mathrm{~mA}$, Reception angle : +/- 45 degrees, VOH ( $\left.6 \mathrm{~V} \mathrm{~V}_{\mathrm{CC}}\right): 4.5 \mathrm{~V}$ and VOL $\left(5 \mathrm{~V} \mathrm{~V}_{\mathrm{CC}}\right): 0.4 \mathrm{~V}$ [9].

\subsection{0x4 LCD Display}

LCD (Liquid Crystal Display) screen is an electronic display module and find and wide range of applications. These modules are preferred over seven segments and other multi segments LEDs. The alphanumeric LCD that we are going to interface is a $20 \times 4$ alphanumeric LCD. It means the LCD can display 20 characters in each row and it has four rows. It is a HD44780 controller based LCD. There are two methods to interface any alphanumeric LCD with Arduino UNO microcontroller: 8-bit and 4-bit interfacing method, only the upper 4 data pins $\left(\mathrm{D}_{4}, \mathrm{D}_{5}, \mathrm{D}_{6}\right.$ and $\left.\mathrm{D}_{7}\right)$ of the alphanumeric LCD are used to send 8-bit data (command) is sent a time using the 8 data lines of the alphanumeric LCD but in 4-bit method, the 8-bit data (command) cannot be sent at a time to the alphanumeric LCD. So, the upper 4 bits of data (command) are sent first and the lower 4-bits are sent later. Figure 3 shows the 20x4 alphanumeric LCD use in this system [7, 8].

\subsection{Relay}

The relay is always configured by using a small driver circuit which consists of a transistor, diode and resistor. Transistor is used to amplify the current so that full current (from the DC source current-9 V battery) can flow through a coil to fully energies it. The resistor is used to provide biasing to the transistor. And diode is used to prevent reverse current flow, when the transistor is switched OFF. Every inductor coil produces equal and opposite EMF when switched OFF suddenly, this may cause permanent damage to components, so diode must be used to prevent reverse current [7, 8]. Six SPDT $+12 \mathrm{~V}$ relays are used in this system. One of them is shown in Figure 3. 


\section{DESIGN AND CONSTRUCTION OF THE SYSTEM}

As shown in Figure 1, the construction of the infrared remote controller for multiple home appliances consists of two parts: the hardware and software implementations. Both software and hardware will be accomplished using Arduino Uno board. This circuit builds around an Arduino Uno board, handheld remote control transmitter, remote control receiver and six channels relays board. System design is based on digital control techniques, all I/O ports are used as only digital ports.

\subsection{Hardware Design}

Schematic diagram of the remote controller for home appliances using Arduino Uno and remote sensor TSOP 1835 is shown in Figure 4. A remote receiver sensor TSOP 1838 is used as the IR sensing device. The output of the TSOP 1838 remote receiver sensor (pin 3) is connected to analog input A0 of Arduino Uno board. Arduino Uno board and relays are powered with a $12 \mathrm{~V} \mathrm{DC}$ adaptor/power source. The output voltage of the $+12 \mathrm{~V} \mathrm{DC}$ adaptor is connected to AN7805 regulator IC's pin1. Output (pin 3) voltage of this IC is applied to the remote receiver sensor pin1 $\left(\mathrm{V}_{\mathrm{I}}\right)$ and $20 \mathrm{x} 4 \mathrm{LCD}$ display's supply pin 2 ( $\mathrm{V}_{\mathrm{DD}}$ ). The different signals from the remote are received by the Arduino Uno microcontroller which then controls the respective relays via a relay driver circuit. The outputs (pin 2 through pin 7) are connected to relays RL1 through RL6 via $10 \mathrm{k} \Omega$ resistors R1 - R6. These relays are used to connect and disconnect the AC loads such that Motor, TV, Fan, Light bulb and more products.

When a button is pressed on the IR remote it sends a sequence of code in form of encoded pulses using $38 \mathrm{kHz}$ modulating frequency. These pulses are received by the TSOP 1838 sensor and then read by the Arduino Uno microcontroller. The controller then decodes the received train of the pulses into a hex values and compares it with the predefined hex values in our program. If any match occurs then the controller performs a relative operation by triggering the respective and the corresponding result is also indicated by 20 x 4 Liquid Crystal Display

Power button on the remote control transmitter is used to determine the all home appliances ON or OFF condition. We have selected key 1 to toggle the relay 1 (Light1), key 2 to toggle the relay 2 (Light 2), key 3 to toggle the relay 3 (Light 3 ), key 4 to toggle the relay 4 (Fan), key 5 to toggle the relay 5 (TV) and key 6 to toggle the relay 6 (Motor).

To trigger the relay we make use of transistors like 2SC1815 which can act as an electronic switch to turn ON/OFF the relays based on the signal from the Arduino microcontroller. The status of the devices can be monitored using $20 \mathrm{x} 4 \mathrm{LCD}$ display. The PCB of the application circuit is drawn by the use of Proteus software with the single sided PCB. The components are placed on the top layer and it is soldered on the bottom layer of the PCB as shown in figure 4.
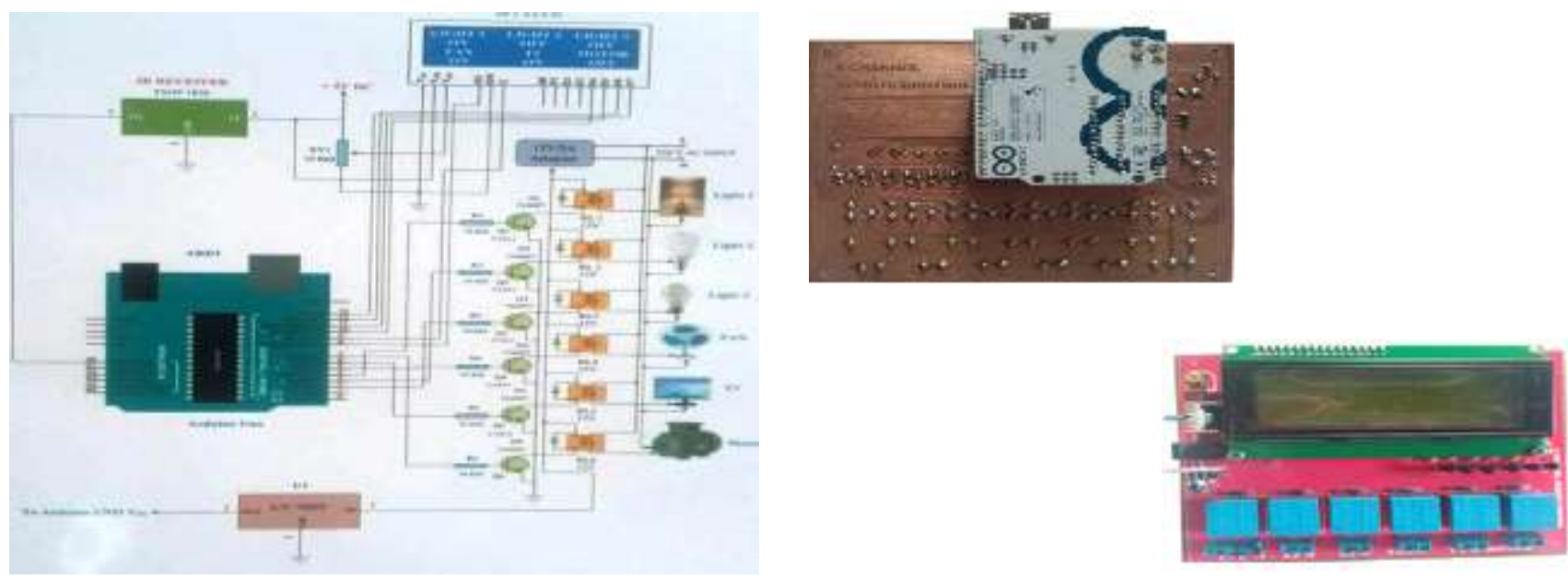

Figure 4. Schematic diagram of the remote controller for home appliance, Bottom view (copper layer) and component view (Top layer)

\subsection{Software Design}

The software program for the Arduino based infrared remote controller for multiple home appliances control system project (IR_Decoder.ino and IR_Remote.ino) is written in Arduino programming language called processing. Arduino Uno is programmed using Arduino IDE software [7] that you can download from arduino.cc. 


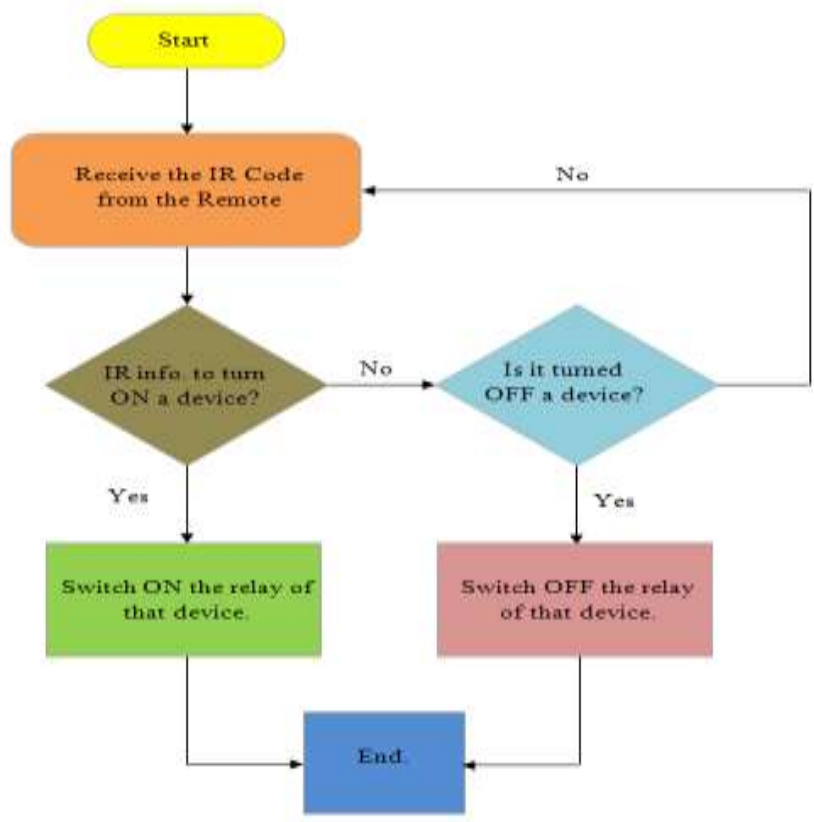

Figure 5. Program flow chart of the Arduino based remote controller for home appliances control system

The program flow chart of the proposed Arduino based remote controller for home appliance control system has been shown in Figure 5 which explains the whole software design procedures. The controller waits for the input from the IR sensor and remote. The remote sends the corresponding train of pulses after the user presses the desired button of the remote. The controller receives the code transmitted from the remote and decodes it in the controller. The received information is decoded form in the first step of the simulation. Once the information is decoded for switching ON or OFF a particular device, the corresponding relay is triggered by the microcontroller and finally the corresponding device is switched ON and OFF using a simple IR remote. Based on the information provided by the user to toggle the device ON or OFF by a single input from IR remote.

As same button is used to toggle the device ON or OFF, the process first checks the present state of the device. Processing of information is done and necessary signal is generated to control the relay associated with the device. The execution state of the device depends on the past state of the device. That is if the device is ON, it will be turned OFF and vice versa. The signal sent to relay by the controller plays a major role in deciding the future state of the device. This is followed across all the components and devices associated in the home automation process. Program window of the propose system is shown in figure 5.

\subsection{Implementation Result and Discussion}

The Circuit created in this project has proved to be functional and has been tested and given results without any error. Operation conditions (on/off stage) of the corresponding appliance are displayed by $20 x 4$ alphanumeric LCD. If any invalid button (not defined in software) on remote is pressed, Arduino Uno board will not response to this action. In this project, six kinds of home appliances are connected to the output of the remote controller system which can operate easily as shown in figure 6 .

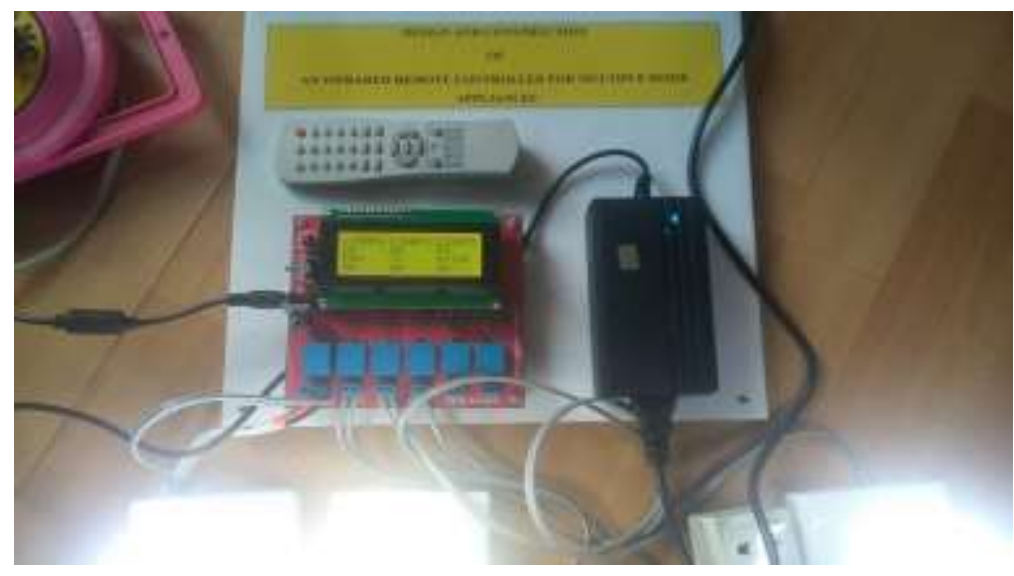

Figure 6. Testing result of remote control system 


\section{CONCLUSION}

This paper presents the construction of Arduino based infrared remote control system which is a device that enables a user to operate or control on-off function of multiple home appliances for desire time. A user only need to select mode from the remote control and system will do the rest of the controlling appliances. This proposed system is highly reliable. This single IR remote controller can be used to manipulate the different kinds of home appliances from nearly 7 meters away. It is compatible for controlling the several home appliances to save the wastage of resources, time and user friendly. This IR remote control circuit is absolutely free from ambient light interference and provides controlling range about 7 meters without the use of any focusing lens. In addition to it can be further control several external devices with modification of programming features to this IR remote control system. This system provides us with extra facility of user friendly, economic benefits and technology updated service to the user.

\section{REFERENCES}

1. Abdulaleem A. Rasheed, Detection and study of various IR handheld remote control signals and using them for home applications, IEEE Conference on Education and e-Learning Innovations (ICEELI), Sousse, 2012.

2. A Roy Delgodo, R. Picking and V.Grout," Remoted-Controlled Home Automation Systems with Different Network Technology", University of Wales, Wrexham, UK, 2013.

3. Dr.Pramod Sharma, PreetiVerma, Km. Bhoomika, Ravi Kumar, SaurabhBaghel, Home Automation Using IR (Infrared) Sensor \& Arduino-Nano Single Board Microcontroller, International Journal of Advanced Research in Electrical, Electronics and Instrumentation Engineering, Vol. 6, Issue 3, March2017

4. C. Woodford, "How remote control and radio control work"8 September 2015 [online] Available http//www.explainthatstuff.com/ remote control html [Accessed 9 April 2016]

5. Hiroshi Kanma, Noboru Wakabayashi, Ritsuko Kanazawa \& Hirimichi Ito, "Home Appliance Control System over Bluetooth with a Cellular Phone", in IEEE, 2003, pp. 1049-1053

6. John Boxall, "Arduino Workshop", 2013.

7. Kaythi Wut Hmone Khin, Khaing Myat Nwe, Lin Lin Phway, Zarni Sann, Ardunio and Bluetooth based Smart Home Control System, International Journal of Scientific Research in Science, Engineering and Technology, (C) 2019, IJSRSET Volume 6, Issue 4

8. T. L. Floyd, Ninth edition, “Digital Fundamentals”, New Jersey, 2006.

9. https://components101.com/sensors/tsop1838-ir-receiver 